\title{
Identification of the main generator source of longitudinal muscle contraction in the earthworm ventral nerve cord
}

\section{Y.C. Chang, Z. Assmé, E.C.L. Caffaro and A.B. Bartoszeck}

\section{Departamento de Fisiologia, U niversidade Federal do Paraná,} Curitiba, PR, Brasil

\section{Correspondence \\ Y.C. Chang \\ Departamento de Fisiologia \\ Setor de Ciências Biológicas, UFPR \\ Caixa Postal 8621 \\ 80011-970 Curitiba, PR \\ Brasil \\ Fax: +55-41-266-2042 \\ E-mail: changyc@cce.ufpr.br \\ Part of a D octoral thesis presented by Z. Assmé to the Departamento de Fisiologia, UFPR, Curitiba, PR, Brasil.}

Received January 20, 1997 Accepted July 27, 1998

\begin{abstract}
The main generator source of a longitudinal muscle contraction was identified as an $\mathrm{M}$ (mechanical-stimulus-sensitive) circuit composed of a presynaptic M-1 neuron and a postsynaptic M-2 neuron in the ventral nerve cord of the earthworm, Amynthas hawayanus, by simultaneous intracellular response recording and Lucifer Yellow-CH injection with two microelectrodes. Five-peaked responses were evoked in both neurons by a mechanical, but not by an electrical, stimulus to the mechanoreceptor in the shaft of a seta at the opposite side of an epidermis-muscle-nerve-cord preparation. This response was correlated to $84 \%$ of the amplitude, $73 \%$ of the rising rate and $81 \%$ of the duration of a longitudinal muscle contraction recorded by a mechanoelectrical transducer after eliminating the other possible generator sources by partitioning the epidermis-muscle piece of this preparation. The pre- and postsynaptic relationship between these two neurons was determined by alternately stimulating and recording with two microelectrodes. Images of the Lucifer Yellow-CH-filled M-1 and M-2 neurons showed that both of them are composed of bundles of longitudinal processes situated on the side of the nerve cord opposite to stimulation. The M-1 neuron has an afferent process (A1) in the first nerve at the stimulated side of this preparation and the M-2 neuron has two efferent processes (E1 and E3) in the first and third nerves at the recording side where their effector muscle cell was identified by a third microelectrode.
\end{abstract}

\section{Introduction}

This series of three papers (the present paper and Refs. 1 and 2) describes a study of the interaction mechanism between substrates in the central and peripheral nervous systems of one familiar yet seldom studied animal, the South American earthworm, Amynthas hawayanus. The mode of this interaction was suggested in a preliminary report (3) showing that the muscle contraction magnitude, i.e., frequency and amplitude, of an isolated epidermis-muscle-nerve-cord (EMNC) preparation is proportional to the size of the remaining tissues after the preparation was dissected. The more tissues left in this preparation, the higher the magnitude of its contractions, be they spontaneous or evoked by a stimulus. Two components are proposed as the neural substrates of this

\author{
Key words \\ - Earthworm muscle \\ contraction \\ - Main generator source \\ $M-1$ and $M-2$ neurons \\ - Mechanical stimulus
}


mechanism. A generator source, possibly located in the central nervous system, evokes this muscle contraction. A modulator which may be in the peripheral nervous system determines the magnitude of this contraction. If this modulation is facilitatory, then the muscle contraction magnitude must be proportional to the amount of peripheral neural substrate left in the preparation. Earthworms have a well-developed peripheral nervous system (4) with several functions proposed (5-7). Our first objective was to identify the neural substrate of a main generator source of muscle contraction and another study (1) was conducted to identify the neural substrate of a modulator with a facilitatory effect on this main generator source. These two neural substrates may either be the known neurons identified by us $(8,9)$ or by others $(10,11)$, or must be identified as new neurons in these two articles. We compared the interaction between these two neural substrates (2) to the conditioning paradigm of the other animals (12-14) and to that of the intact earthworm $(15,16)$. This comparison is expected to contribute to the knowledge of learning and memory mechanism in general.

\section{Material and Methods}

Mature South American earthworms identified as Amynthas hawayanus and raised in a terrarium outside the laboratory were used in this study. Only the middle body between the 25th and 70th segments was used for their repeated neuronal organization (17) important for reproducible results. Dissection of the EMNC (Figure 1), electrophysiological equipment and experimental procedures were described in previous articles $(8,9)$.

\section{Stimulation and recording}

Stimuli were square pulses delivered from a CURITIBA-1 stimulator (manufactured by the authors of this article, Curitiba, PR, Brazil). A mechanical stimulus (MS; Figure 1) was delivered through a solenoid-driven stylus perpendicular to a seta shaft (S; Figure 1) at the flipped-up left posterior corner of this preparation in order to bend this seta to stimulate the mechanoreceptor inside it (9). An electrical stimulus was delivered through a glass tube suction electrode to the muscle surface of the left side of this preparation, since it was found that an electrical stimulus to its epidermal surface did not always evoke a response in neurons and muscle cells. The intensity of the electrical stimulus was read from the stimulator dial but the intensity of the mechanical stimulus varied according to the distance and angle of this stylus from the seta shaft and according to the different pliability of each seta. By aligning the stylus and the seta as consistently as possible in each test and by choosing similar setae each time, the number of responding muscle fibers increased proportionally to the increasing stimulator dial values from 1 to 20 volts and from 1 to $20 \mathrm{~ms}$ until responses could be recorded from all muscle fibers over the entire surface of the preparation. The minimal number of muscle fibers responding to the minimal intensity of a stimulus was considered as the effector muscle cell into which Lucifer Yellow-CH (L 0259, Sigma Chemical Co., St. Louis, MO) was injected. It was seen as a longitudinal bundle of processes near the exit of an efferent nerve into the muscle layer contralateral to the stimulus (E; Figure 7 in Ref. 1). This minimal intensity of mechanical stimulus was always between 35 volts and 5-10 ms on the stimulator dial. When two stimuli were used, the intertrial interval (ITI) and interstimulus interval (ISI) could be adjusted from $+200 \mathrm{~ms}$ to $-200 \mathrm{~ms}$ by a built-in synchronizer in this stimulator.

Only the response evoked by the delivered stimuli was recorded (Figure 2) although spontaneous electrical (N; Figure 2-1) and mechanical (i.e., contractions, C) (Figure 21) activities were also monitored. Extracel- 
lular electrical responses were recorded from the segmental nerves (N; Figure 2) by suction electrodes on a Tektronix 5113 oscilloscope (Beaverton, OR). Intracellular electrical responses were recorded from neurons and muscle cells by microelectrodes (AM, 6010, Everett, USA) filled with 7\% Lucifer Yellow-CH on another trace of this oscilloscope. A high-impedance amplifier bridge (Model 8500, Dagan Corp., Minneapolis, $\mathrm{MN}$ ) enabled each of these microelectrodes to be used for recording, stimulation and dye-injection simultaneously. These microelectrodes were mounted on separate micromanipulators (Model CS-56-3, Custom Instruments, Whippany, USA) to facilitate the search for neurons and muscle cells by independent scanning over the entire surface of this preparation. Mechanical responses, i.e., muscle contractions (C; Figure 2 ), were recorded with a mechano-electrical transducer. Although such transducers are available commercially, or can be constructed on the basis of literature data (e.g., 18), a low-damping device was designed to preserve the rising and falling rates of a contraction as much as possible. The device consisted of a photographic photocell (Metrawatt, AG, Nürenberg, Germany) (Phc; Figure 1) with a small light spot shining on it from a battery-operated (for reducing 60cycles interference with the records) toy torch (T; Figure 1). The amount of light received by this photocell was controlled by the movement of an aluminum foil cover (Alc; Figure 1) pulled by the muscle contraction. One end of this aluminum foil cover was connected to the anterior end of the right side of this preparation and the other end to a small spring. By adjusting this spring, the movement of this aluminum foil cover could be made linearly proportional to the amount of light it allowed to pass to the photocell in a range of 0.1 to $5.0 \mathrm{~g}$ of force on the Tektronix 5113 oscilloscope trace (Osc; Figure 1). Notice that the mechanical friction of this setup was low because the two connecting

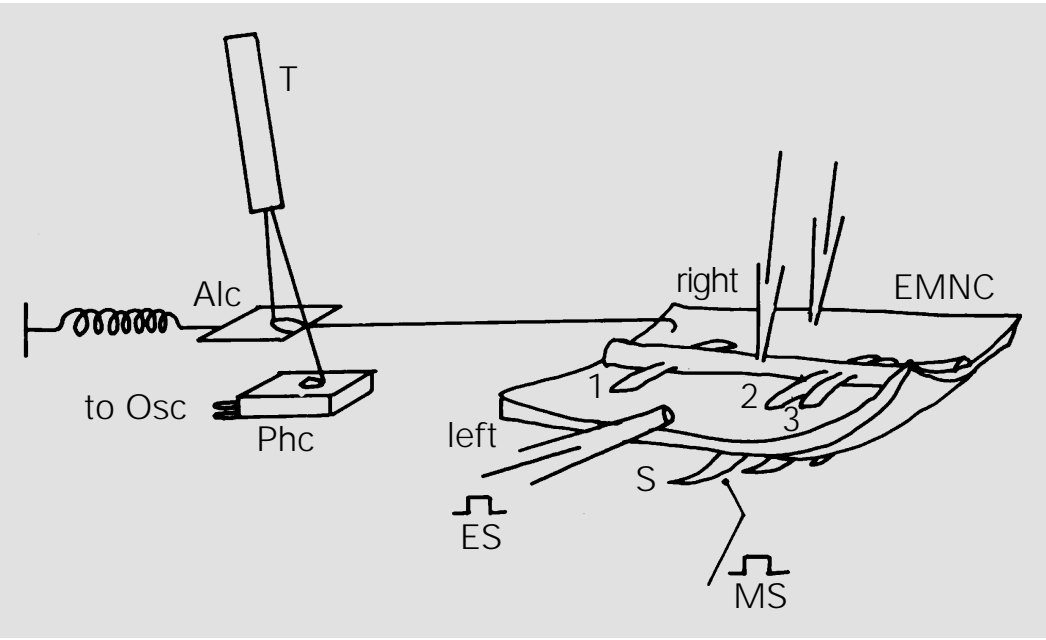

Figure 1 - EMNC, Epidermis-muscle-nerve-cord preparation. The inner side is toward the reader, anterior end to the left, with two microelectrodes (actual number, four) for stimulation and recording, one suction electrode for delivering extracellular electrical stimulation (ES) and one stylus for delivering mechanical stimulation (MS) to a seta (S) at the flipped-up left posterior corner of this preparation. Three pairs of segmental nerves are indicated by numbers. The transducer assembly of mechanical recording of muscle contraction is shown on the left side of this figure. T, Battery-operated toy torch; Alc, aluminum foil cover; Phc, photocell; Osc, oscilloscope. Structures in this figure are not drawn to scale.

lines at both ends of this aluminum foil cover were suspended in the air without contacting any solid object. Both the velocity (in straight lines) and acceleration (in curves) of these rising and falling rates (in $\mathrm{g} / \mathrm{s}$ ) of a muscle contraction were preserved as much as possible by this design. In addition to the rising and falling rates, the amplitude (in grams) and the duration (in seconds) at 2/3 amplitude of muscle contraction were also recorded and measured for comparison.

\section{Isolation of the main generator source of muscle contraction}

Four systems are known to be capable of generating contraction in an earthworm muscle cell. They are the central nervous system $(2,5-7,11)$, the peripheral nervous system $(2,7)$, the muscle system which transmits impulses through their nexi $(5,7,19)$ and the spontaneous activation in a single muscle cell membrane (20). The experiments in this section were carried out to determine which of them is the "main" generator source 


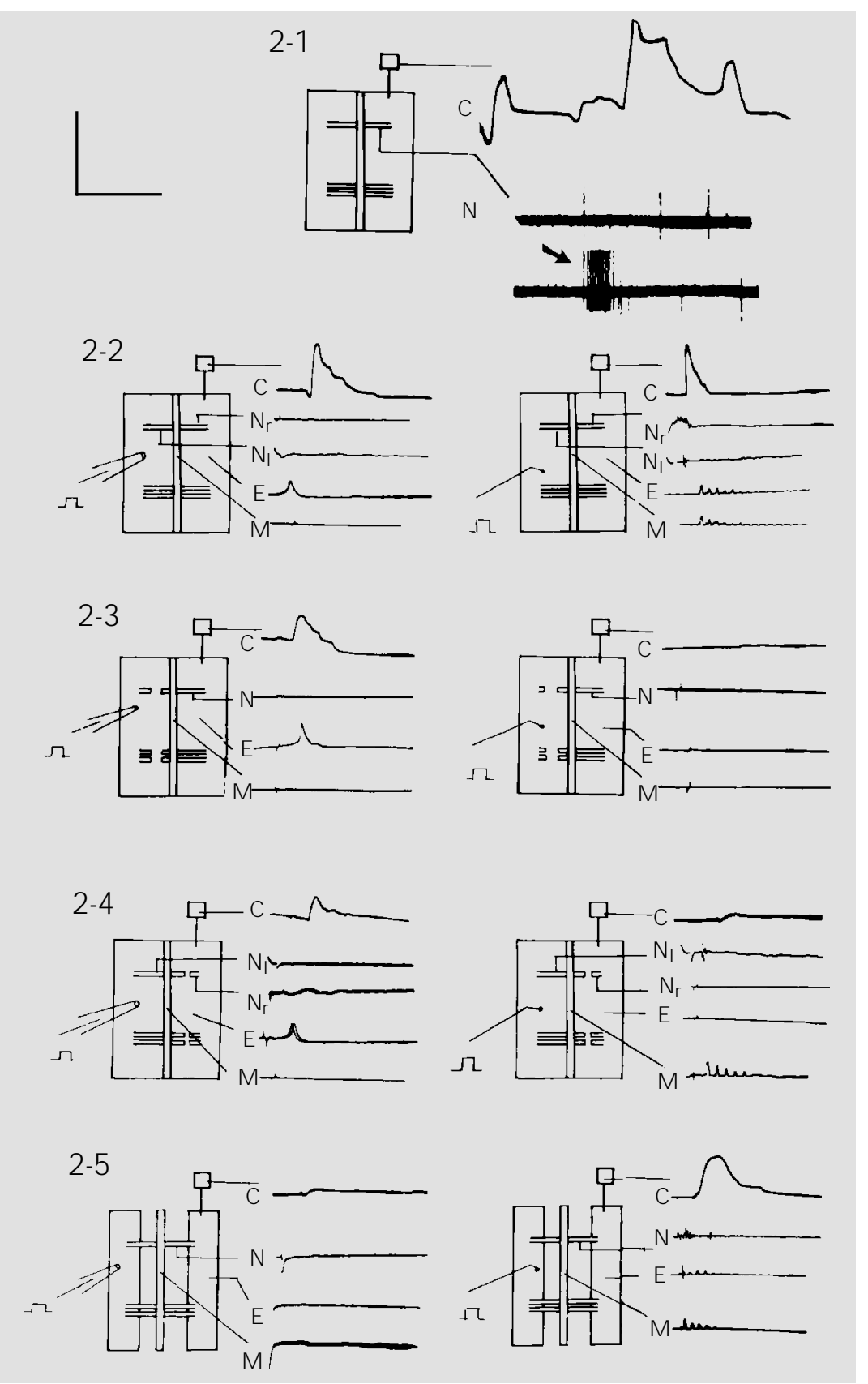

Figure 2 - Neuron and muscle cell activities.

2-1, Spontaneous activities of an intact EM NC preparation without stimulation.

2-2, Responses of an intact EMNC preparation evoked by electrical (left column) and mechanical (right column) stimuli.

2-3, Responses of an EMNC preparation evoked by electrical and mechanical stimuli after section of all afferent (left) segmental nerves.

2-4, Responses of an EMNC preparation evoked by electrical and mechanical stimuli after section of all efferent (right) segmental nerves.

2-5, Responses of an EMNC preparation evoked by electrical and mechanical stimuli after section, or partition, of the epidermis-muscle piece.

$C$, Contraction; $N$, segmental nerves; $N_{l}$, left segmental nerve; $N_{r}$, right segmental nerve; $E$, effector muscle response; $M$, mechanical stimulus-sensitive neuron response in the nerve cord. Calibration: $\mathrm{C}, 5 \mathrm{~g}$ and $5 \mathrm{~s} . \mathrm{N}, \mathrm{N}_{\mathrm{l}}$, and $\mathrm{N}_{\mathrm{r}}, 5 \mathrm{mV}$ and $50 \mathrm{~ms}$. $\mathrm{E}$ and $\mathrm{M}, 50 \mathrm{mV}$ and $50 \mathrm{~ms}$. which generates the largest percentage of the muscle contraction and to isolate it by differential sectioning (Figure 2). Contractions evoked by peripheral electrical (left column; Figure 2) and mechanical (right column; Figure 2) stimuli were used to identify these sources. Two kinds of differential sectioning were done in these experiments. Sectioning the afferent (Figure 2-3), or the efferent (Figure 2-4), segmental nerves was performed to interrupt the transmission routes through the central nervous system. Sectioning, or partitioning, the epidermis-muscle piece (Figure 2-5) was performed to interrupt the transmission routes through the peripheral nervous system, the muscular system and the single muscle cell. While isolating the central circuits, it must be kept in mind that all segmental nerves contain afferent and efferent fibers. In order to isolate the afferent from the efferent fibers, one must sacrifice the reflex arcs with ipsilateral afferent and efferent routes and to preserve only the arc with the contralateral routes. This was done by stimulating only the left side of this preparation and recording only from the right side as shown in Figures 1 and 2. All responses recorded from the left side nerves, $\mathrm{N}_{\mathrm{l}}$, were then considered as purely afferent and all responses recorded from the right side nerves, $\mathrm{N}_{\mathrm{r}}$, were purely efferent in this arrangement (21) although the opposite would be equally valid because the body plane is bilaterally symmetrical in this animal.

\section{Identifying the neuron circuit of the main generator source}

The pre- and postsynaptic relationship between two neurons in the mechanicalstimulus-sensitive circuit (abbreviated as the M-circuit) in the nerve cord was determined by alternately stimulating and recording with two microelectrodes (Figure 3). There may be three situations of transmission between two neurons: "no transmission", where excitation of either one of them did not evoke a 
response in the other, "rectifying, or orthodromic, transmission", where excitation in a presynaptic M-1 neuron evoked a response in a postsynaptic M-2 (Figure 3-1, right) neuron but excitation in an M-2 neuron did not evoke a response back in the M-1 neuron (Figure 3-1, left), and "non-rectifying transmission" where excitation in either one of them evoked a response in the other. Only the second situation, or "rectifying transmission", was further studied in this article. The third situation, or "non-rectifying transmission", may also be authentic between some of these neurons but was not studied because of the difficulty in further confirmation. The diffusion of Lucifer Yellow-CH (22) injected into one of these neurons was also tested for its rectifying or non-rectifying properties. Another characteristic which may also help distinguish the M-1 and M-2 neurons is their possible different connection to other neurons outside their own circuit. Their different connections to higher center intersegmental neurons were tested by stimulating the anterior end of the nerve cord and recording its response from these two neurons (Figure 3-2).

\section{Results}

\section{Spontaneous activities}

Spontaneous activities are the electrical discharges from the segmental nerves (N; Figure 2-1) and muscle contractions (C; Figure 2-1) not correlated to the delivered stimulus. These two activities were found not to be correlated, i.e., they do not seem to evoke a response from each other. Both of them have irregular frequencies, amplitudes, rising and falling rates and durations. Some of these spontaneous contractions had no measurable duration because they did not return to baseline $(\mathrm{C}$; Figure 2-1). The spontaneous electrical discharges from the segmental nerves had irregular intervals but maintained an average

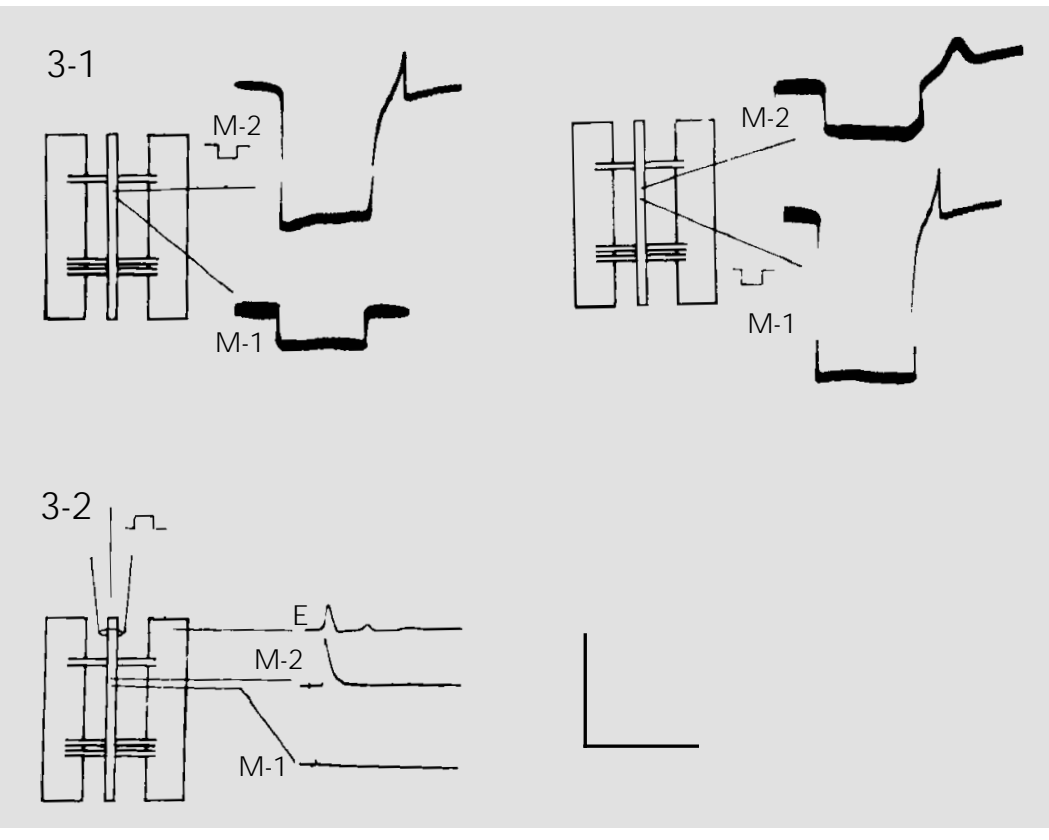

Figure 3 - Synaptic connection between the main generator neurons.

3-1, Rectifying synaptic transmission demonstrated by alternate intracellular stimulation with hyperpolarizing 7.0-nA, 50-ms pulses. Notice that a postsynaptic (M-2) response evoked by this stimulus is not transmitted to the presynaptic $(M-1)$ neuron in the left figure while a presynaptic $(M-1)$ response evoked by this stimulus is transmitted to the postsynaptic $(M-2)$ neuron in the right figure.

3-2, Different connections to higher intersegmental centers examined by extracellular depolarizing pulse to the anterior end of the nerve cord. Only postsynaptic (M-2) neuron has connection to the higher center. Presynaptic (M-1) neuron does not respond to this stimulus.

E, Effector muscle response. Calibration: $50 \mathrm{mV}$ and $50 \mathrm{~ms}$ for both figures. frequency of $0.74 \pm 0.21 \mathrm{~Hz}$ in 50 preparations measured for $30 \mathrm{~min}$ each (top line $(\mathrm{N})$; Figure 2-1). There were also high frequency bursts without correlation with any noticeable events (arrow, bottom line; Figure 2-1). The resting potentials of both muscle cells and neurons decreased and increased unpredictably between 30 and 80 $\mathrm{mV}$ when recorded continuously for longer than $1 \mathrm{~min}$. Spontaneous discharges of these cells might or might not accompany these resting potential fluctuations. These spontaneous activities, although part of behavior, were not studied in the present investigation. Only responses evoked by the delivered stimuli were studied in the following sections. 


\section{Effector responses}

Effector responses are the muscle contractions (C; Figure 2-2) and electrical responses recorded from the muscle cells $(\mathrm{E}$; Figure 2-2) and from the nerves $\left(\mathrm{N}_{\mathrm{r}}, \mathrm{N}_{\mathrm{l}}\right.$; Figure 2-2) on one side (right) of this preparation evoked by a mechanical, or electrical, stimulus on the other side (left). The contractions evoked by either electrical $(\mathrm{C}$, left column; Figure 2-2), or mechanical (C, right column; Figure 2-2) stimuli in an intact EMNC preparation were similar. Three parameters were measured from 50 muscle contractions in 23 preparations, i.e., amplitude $(2.15 \pm 0.19 \mathrm{~g})$, rising rate $(8.72 \pm 0.16$ $\mathrm{g} / \mathrm{s})$ and duration at $2 / 3$ amplitude $(0.31 \pm$ $0.11 \mathrm{~s}$ ).

The electrical responses evoked in the effector muscle cell by the mechanical and electrical stimuli were different. Those evoked by the electrical stimuli were singlepeaked (E, right column) but those evoked by the mechanical stimuli were five-peaked (E, left column).

\section{Main generator source}

Although both electrical (left column; Figure 2-2) and mechanical (right column; Figure 2-2) stimuli evoked muscle contractions, $\mathrm{C}$, on the opposite side of this preparation, the electrical stimulus did not evoke an afferent response in the left segmental nerves, $\mathrm{N}_{1}$, or in the neurons in the nerve cord, but the mechanical stimulus evoked both afferent, $\mathrm{N}_{1}$, and efferent, $\mathrm{N}_{\mathrm{r}}$, nerve responses and five-peaked responses, $\mathrm{M}$, in many neurons in the nerve cord.

Section of all afferent (left) segmental nerves (Figure 2-3) did not change the effector muscle cell electrical response, E, and contraction, C, evoked by the electrical stimulus (left column) but eliminated the efferent nerve response, $\mathrm{N}_{\mathrm{r}}$, central neuron response, $\mathrm{M}$, and muscle cell electrical, E, and mechanical, $\mathrm{C}$, responses evoked by the me- chanical stimulus (right column). These two experiments (Figure 2-2 and 2-3) showed that the responses evoked by the electrical stimulus were not transmitted through the neurons in the nerve cord to reach the effector muscle cell. Only the response evoked by the mechanical stimulus was transmitted through the neurons in the nerve cord to reach the effector muscle cell.

Section of all efferent nerves (right) did not attenuate much of the muscle contraction, $\mathrm{C}$, evoked by the electrical stimulus (left column; Figure 2-4) but reduced significantly the amplitude evoked by mechanical stimuli (right column; Figure 2-4) to $38 \%$, or $0.81 \pm 0.14 \mathrm{~g}$, and the rising rate to $25 \%$, or $2.21 \pm 0.33 \mathrm{~g} / \mathrm{s}$, and increased its duration at $2 / 3$ amplitude to $225 \%$, or $0.71 \pm 0.21 \mathrm{~s}$ in 50 contractions. Section of the suspected transmission route of the electrical stimulusevoked response by partitioning the epidermis-muscle piece along its midline while leaving all segmental nerves intact (Figure 25) almost eliminated, but never completely, the muscle contraction, $\mathrm{C}$, evoked by electrical stimulus (left column) but only reduced non-significantly the amplitude to $84 \%$, or $1.85 \pm 0.13 \mathrm{~g}$, and the rising rate to $73 \%$, or $6.35 \pm 0.46 \mathrm{~g} / \mathrm{s}$, and increased the duration at $2 / 3$ amplitude to $123 \%$, or $0.38 \pm 0.10 \mathrm{~s}$, equivalent to a reduction of $81 \%$, in 50 contractions evoked by the mechanical stimulus (right column). The last three percentages were believed to be the percentages of a muscle contraction evoked by the mechanical stimulus alone without the nonsignificant contribution from the electrical stimulus.

\section{Synaptic connection between the main generator neurons}

Two microelectrodes were inserted into two mechanical-stimulus-sensitive neurons in the nerve cord. An intracellular electrical stimulus was delivered through one of these microelectrodes while the other recorded the 
response transmitted from the stimulated neuron. As these neurons hardly responded to an intracellular depolarizing electrical stimulus, a hyperpolarizing (square pulse symbol in Figure 3-1 and 3-2) 50-ms pulse slightly above the threshold value (7.0 nA) was always used to evoke an off-response in the form of a single-peaked action potential in one of these neurons (M-2; Figure 3-1). If no response was evoked in the next neuron (M-1; Figure 3-1, left) except for the spreading square pulse, stimulation and recording were switched around in these two microelectrodes (Figure 3-1, right). If the first neuron did not respond to the response in the second, this neuron pair was discarded as "no transmission", i.e., they do not form a circuit. Only when the postsynaptic neuron (M-2) did not evoke a response in the presynaptic neuron (M-1; Figure 3-1, left) but the presynaptic neuron evoked a response in the postsynaptic neuron (M-2; Figure 3-1, right) was this pair accepted as forming a rectifying two-neuron circuit. Thirty-one of the identified 42 pairs (62 neurons among 84) satisfied this criterion. Non-rectifying pairs which responded in both ways were also not used in this study because they could not be further distinguished as to whether they were composed of only one, or two, neurons. On the other hand, diffusion of Lucifer Yellow$\mathrm{CH}$ seemed to be always non-rectifying from one neuron to the next (22) in this circuit but never to a third neuron. The last test confirmed that this generator source was formed by a two-neuron circuit.

When the ventral nerve cord anterior to the identified neuron pair was stimulated extracellularly with a suction electrode (Figure 3-2), none of these 31 identified M-1 neurons responded but $26 \mathrm{M}-2$ neurons in 13 pairs responded. This additional characteristic showed that only the postsynaptic, but not the presynaptic, neuron was commanded by a higher intersegmental center and was also used for their identification. Thus, only the last 13 pairs were physiologically identi- fied as the main generator source.

\section{Morphology of the main generator source}

Nine of the 13 physiologically identified neuron pairs (18 of the 26 neurons) showed consistent morphology when their Lucifer Yellow-CH-filled images were examined under the fluorescent microscope. Two of the most typical examples are shown in Figure 4 of the present paper and Figure 7 of Ref. 1 . The most visible structures in the whole-mounting preparation viewed in the radial direction (WM; Figure 4R) (9) are two parallel bundles of longitudinal processes separated by a distance of no more than 100 $\mu \mathrm{m}$ and a group of varicosities (V; Figure $4 \mathrm{R}$ ) on the right side and in the dorsal quadrant of the ventral nerve cord when viewed in the anteroposterior direction (Figure 4AP). A thin process with a series of curves shorter than $30 \mu \mathrm{m}$, a unique characteristic of neuron process $(8,9)$, can be seen coming in from the ipsilateral (left) first nerve (A1; Figure 4R) to the medial longitudinal bundle, and two thin processes can also be seen going out of the lateral longitudinal bundle through the contralateral (right) first (E1; Figure 4R) nerves. These are the afferent and efferent fibers with their responses recorded as $\mathrm{N}_{1}$ and $\mathrm{N}_{\mathrm{r}}$ in Figure 2. The somata of these two neurons may be too small to be distinguished from these structures. Although branches of these two longitudinal bundles approach each other in several places, actual synapses between them are not visible. Comparing this morphology to the physiological records, the medial longitudinal bundle of the process corresponds to the presynaptic neuron (M-1; Figure 4R, WM) (also visible in the middle of Figure 4R, V) and the lateral bundle to the postsynaptic neuron (M-2; Figure 4R, WM) (also visible in the middle of Figure 4R, V) in this circuit. The entire system is tentatively called the contralateral M-circuit, or simply the M-circuit, in this and in the two following articles $(1,2)$. 
Figure 4 - Morphology of the main generator source. Fluorescence microscope photograph and camera-lucida drawing of Lucifer Yellow-CH-filled images. $\mathrm{R}$, Radial dimensional view; WM, whole-mounting preparation; $A 1$, afferent process in the first nerve of the stimulated (left) side of this preparation; E1, efferent process in the first nerve of the recording (right) side of this preparation; $\mathrm{E} 3$, efferent process in the third nerve of the recording (right) side of this preparation; $\mathrm{V}$, varicosities in the central region of these identified neurons; $M-1$, presynaptic neuron; $M-2$, postsynaptic neuron. Two longitudinal bundles of processes representing these two neurons are also visible in the upper middle region of $\mathrm{V}$. AP, Anteroposterior view of the same preparation at a level near $\mathrm{V}$. Some of the branches extend dorsoventrally and are not detectable in the $\mathrm{R}$ view above Calibration: $100 \mu \mathrm{m}$ for all figures.
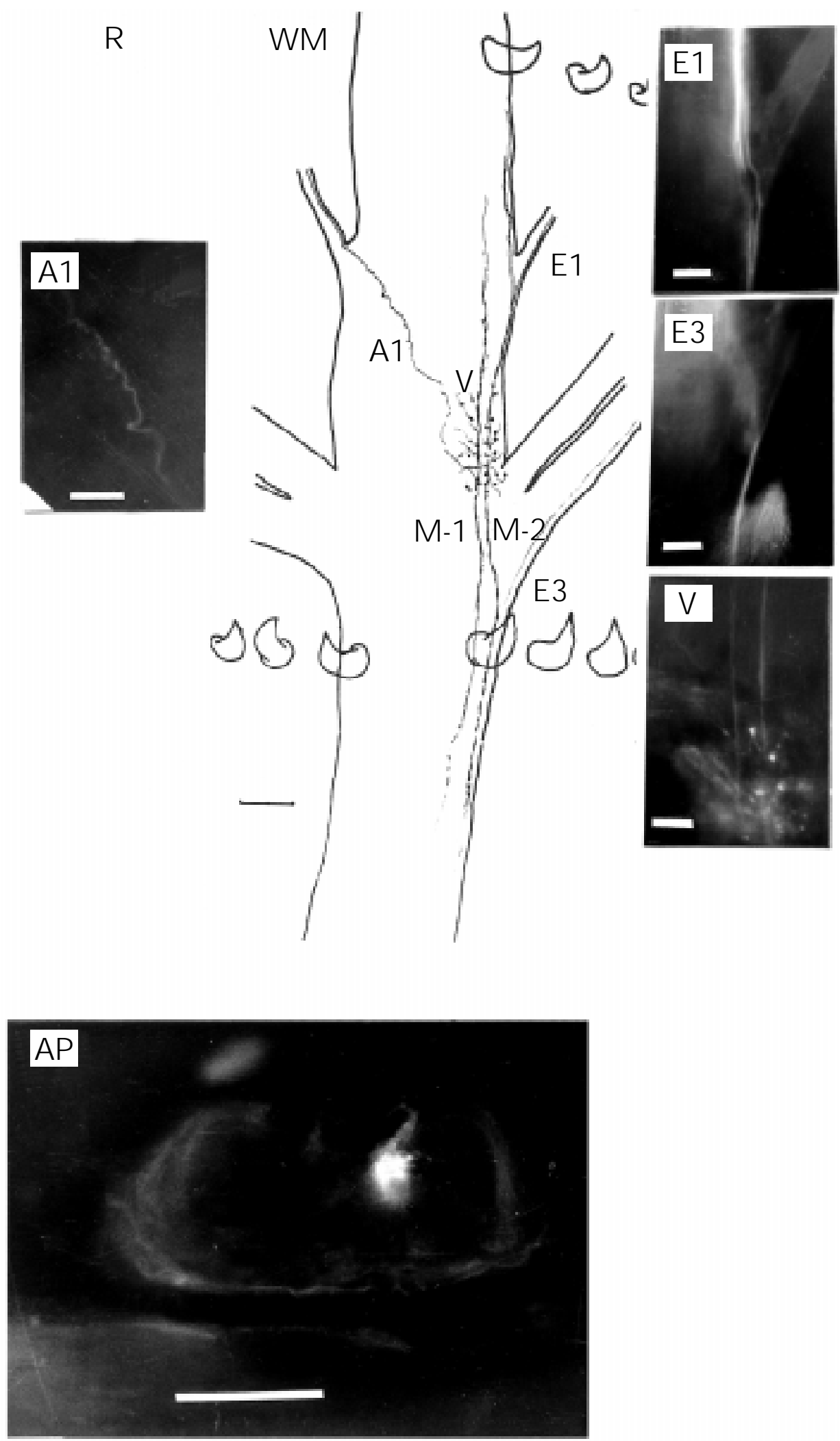


\section{Discussion}

Only 18 neurons identified in nine preparations (one pair in each) were accepted as the main generator source in the present study although a total of 84 neurons in 42 preparations satisfied the primary criterion that their five-peaked responses were evoked by a threshold mechanical stimulus to the setal mechanoreceptor and their responses were correlated to the electrical responses and contractions of the contralateral muscle cell. These nine neuron pairs were selected because of their physiological and morphological consistency. Circuits with variations were rejected in this procedure. Most of these variations were related to their synaptic transmission mechanism. Only rectifying pre-(M-1) and post-(M-2) synaptic neurons in an M-circuit were accepted. It was suspected that many of them had non-rectifying transmission. They were rejected because their pre- and postsynaptic relationship could not be determined and because one could not distinguish morphologically if they were composed of a single neuron or two. On the other hand, all these neurons were non-rectifying to Lucifer Yellow-CH diffusion (22). Those having rectifying diffusion were rejected because only one neuron was morphologically visible. Therefore, this high rejection rate was the result of a predetermined bias of the experimenters. Actual two-neuron Mcircuits for generating $70-80 \%$ of a contraction in the contralateral effector muscle cell must be more numerous than the nine pairs reported here. Slight physiological or morphological variation may actually be the rule rather than the exception. This predetermined bias of selection was nevertheless upheld in this article because a precise quantitative measurement was a necessity for comparing the muscle contraction magnitude modifications in the two subsequent studies $(1,2)$. But this predetermined bias in their isolation and identification imposed a limit on the comparability of the results of this article to the stimulus-response paradigm in intact preparations tested by experimental psychological methods $(15,16)$, where not only all segmental neuronal and muscular circuits but also intersegmental circuits from the highest to the lowest centers are included. In addition, one must also not overlook the interpretation of one single stimulus, such as that of a chemical, into a mixture of mechanical and thermal stimuli, by the conscious subject in experimental psychology. This limit in comparability may be mitigated by adding more factors in future experiments, such as a study of the Q (chemical-stimulus-sensitive) circuit (23), but cannot be addressed in the preliminary experiments reported in the present article.

\section{References}

1. Chang YC, Assmé $Z$ \& Bartoszeck $A B$ (1998). Facilitation of the main generator source of earthworm muscle contraction by a peripheral neuron. Brazilian J ournal of Medical and Biological Research, 31: 1295-1302.

2. Chang YC, Caffaro ECL, Assmé $Z$ \& Bartoszeck AB (1998). Persistent attenuation and enhancement of the earthworm main muscle contraction generator response induced by repeated stimulation of a peripheral neuron. Brazilian J ournal of Medical and Biological Research, 31: 1303-1311.
3. Chang YC \& Assmé Z (1989). Neurônios no circuito reflexo periférico da minhoca, Amynthas hawayanus. IV Reunião Anual da Federação de Sociedades de Biologia Experimental, Caxambu, MG, Brasil, 21 (Abstract).

4. Zyeng DH (1930). Distribution of intermuscular nerve cells in the earthworm. Tohoku Imperial University Science Reports, Series 4, 5: 449-466.

5. Prosser CL (1934). The nervous system of the earthworm. Quarterly Review of Biology, 9: 181-200.

6. Prosser CL (1946). The physiology of ner- vous systems of invertebrate animals. Physiological Reviews, 26: 337-382.

7. Gardner CR (1976). Neuronal control of locomotion in earthworm. Biological Reviews, 51: 25-52.

8. Chang YC \& Assmé $Z$ (1989). Identification of neurons involved in the earthworm, Amynthas hawayanus, reflex activity. Comparative Biochemistry and Physiology, Section A, 92: 171-179.

9. Chang $Y C, M$ archioro $M \&$ Assmé $Z$ (1991). Two kinds of peripheral afferent neurons in the earthworm reflex arc. Comparative Biochemistry and Physiolo- 
gy, Section A, 100: 563-569.

10. Günther J (1972). Giant motor neurons in the earthworm. Comparative Biochemistry and Physiology, Section A, 42: 967973.

11. Drewes CD (1984). Escape reflexes in earthworm and other annelids. In: Eaton RC (Editor), Neural Mechanism in Startle Behavior. Plenum Press, New York, 4391.

12. Carew TJ , Walters ET \& Kandel ER (1981). Classical conditioning in a simple withdrawal reflex in Aplysia californica. J ournal of Neuroscience, 1: 1426-1437.

13. Lukowiak K \& Sahley C (1981). The in vitro classical conditioning of the gill withdrawal reflex of Aplysia californica. Science, 212: 1516-1518.

14. Sastry BR, Goh J W \& Auyeung A (1986). Associative induction of posttetanic and long-term potentiations in CA1 neurons of rat hippocampus. Science, 232: 988990.

15. Ratner SC \& Miller KR (1959). Classical conditioning in earthworms, Lumbricus terrestris. Journal of Comparative and Physiological Psychology, 52: 102-105.

16. Abramson $\mathrm{Cl} \&$ Buckbee DA (1995). Pseudoconditioning in earthworms (Lumbricus terrestris): Support for nonassociative explanation of classical conditioning phenomena through an olfactory paradigm. J oumal of Comparative Psychology, 109: 390-393.

17. Smith PH \& Mittenthal J E (1980). Intersegmental variations of afferent pathways to giant interneurons of the earthworm, Lumbricus terrestris L. Comparative Biochemistry and Physiology, Section A, 140: 351-363.

18. Drewes CD \& Pax RA (1971). Mechanical response of body wall muscle of the earthworm, Lumbricus terrestris, to segmental nerve stimulation. Canadian J ournal of Zoology, 49: 1527-1534.

19. Belestin BE (1982). Membrane physiology of excitable cells in Annelids. In: Podesta RB (Editor), Membrane Physiology of Invertebrates. Marcel Dekker, New York, 199-260.
20. Chang YC (1969). Membrane potential of muscle cells from the earthworm, Pheretima hawayana. American J oumal of Physiology, 216: 1258-1265.

21. Assmé Z (1983). Controle reflexo dos músculos somáticos de Amynthas hawayanus (Rosa, 1891) (Megascolecidae, Oligochaeta). Master's thesis, Universidade Federal do Paraná, Curitiba, PR, Brasil.

22. Brink PR \& Ramanan SV (1985). A model for the diffusion of fluorescent probes in the septate giant axon of earthworm. Biophysical J ournal, 48: 299-309.

23. Chang YC, Assmé $Z \&$ Bartoszeck $A B$ (1996). Distribuição de frequências da resposta aferente evocada pelo estímulo químico periférico na minhoca. IV. Neurônios centrais que respondem aos estímulos químicos na minhoca. XI Reunião Anual da Federação de Sociedades de Biologia Experimental, Caxambu, MG, Brasil, 28 (Abstract). 